\title{
Tolerance of Ten Lettuce Cultivars to High Temperature Combined with $\mathrm{NaCl}$ during Germination
}

Janice M. Coons

Botany Department, Eastern Illinois University, Charleston, IL 61920

Robert O. Kuehl

Statistical Support Unit, Department of Agricultural Economics, University of Arizona, Tucson, AZ 85721

Nancy R. Simons

Department of Plant Sciences, University of Arizona, Tucson, AZ 85721

Additional index words. Lactuca sativa, heat stress, salt stress, crisphead lettuce, leaf lettuce

\begin{abstract}
Water that may contain salt often is used to cool soil to help overcome high-temperature inhibition of lettuce germination. This study was done to determine how lettuce cultivars differ in their germination response to high temperature and $\mathrm{NaCl}$. Ten lettuce (Lactuca sativa L.) cultivars (Grand Rapids, Climax, Coolguard, Empire, Great Lakes 659-700, Mesa 659, Salinas, Vanguard 75, Red Coach 74, and Wintersupreme) were germinated at 20, 25,30 , or $35 \mathrm{C}$ with $0.0,-0.3,-0.6,-0.9,-1.2$, or $-1.5 \mathrm{MPa} \mathrm{NaCl}$. With no NaCl, germination percentages and rates decreased significantly at $35 \mathrm{C}$ for all cultivars except 'Salinas', which decreased at 30C. With higher concentrations of $\mathrm{NaCl}$, decreases in germination percentages and rates were observed at lower temperatures. Cultivar differences in response to temperature were present with no $\mathrm{NaCl}$ but became larger in the presence of NaCl. 'Great Lakes 659-700' and 'Mesa 659' were most sensitive to high temperature and salt. 'Coolguard' and 'Empire' were most tolerant to high temperature and salt, with some tolerance also present in 'Grand Rapids' and 'Vanguard 75'. Relative tolerance of cultivars to $\mathrm{NaCl}$ as shown by germination percentages and rates was consistent with growth of roots.
\end{abstract}

Lettuce seed germination is inhibited by soil temperatures in excess of 25 to $32 \mathrm{C}$, depending on the cultivar (Borthwick and Robbins, 1928; Gray, 1975; Thompson et al., 1979). These temperatures often are exceeded when lettuce is planted during late August or September for winter production in southwestern states, such as Arizona and California (Valdes and Bradford, 1987). Germination often is poor, resulting in nonuniform stand establishment and subsequent variability in maturity at harvest (Gray, 1975; Krause, 1980; Wurr and Fellows, 1983). In an attempt to overcome this problem, growers frequently keep the soil surface moist until stands are established. Although this practice helps reduce soil temperature, salts dissolved in the irrigation water also are applied to the soil surface. Much irrigation water in southwestern states measures around 0.0 to -0.3 $\mathrm{MPa}$, although some measures as high as - 0.6 MPa (Dutt and McCreary, 1970). Salts in the upper few centimeters of the soil may be as high as $14 \mathrm{mmhos} \cdot \mathrm{cm}^{-1}$ (J. M. C., unpublished).

We found no studies in the literature considering differences in response of lettuce cultivars to combinations of high temperature and salt. Some studies dealt with response of lettuce to salt only. Lettuce is moderately sensitive to salt relative to other species (Maas and Hoffman, 1977), with some researchers reporting differences in sensitivity among cultivars (Pasternak et al., 1986; Shannon and McCreight, 1984; Shannon et al., 1983) and others reporting no differences (Ayers et al., 1951). However, seedlings frequently were started with fresh water and treated with salt solutions later. In some studies, seeds of various lettuce cultivars were imbibed in salt solutions, although

Received for publication 22 June 1989. Work supported by the Univ. of Arizona Agricultural Experiment Station. We gratefully acknowledge Rick Axelson for his assistance with statistical analysis and Laura Summerfield for her assistance in the root length experiments. The cost of publishing this paper was defrayed in part by the payment of page charges. Under postal regulations, this pape therefore must be hereby marked advertisement solely to indicate this fact. only one concentration of salt was tested with no control of temperature (Shannon and McCreight, 1984; Shannon et al., 1983). Only one study was found that considered how $\mathrm{NaCl}$ and temperature affected lettuce during germination and early seedling growth (Odegbaro and Smith, 1969). They found that salt decreased seedling fresh weight and that high temperature accentuated this effect, but only one cultivar was tested.

Due to limited studies on the effect of high temperature and salt during lettuce germination, we determined how germination percentages and rates of 10 lettuce cultivars differ in response to high temperature and $\mathrm{NaCl}$, and considered how seedlings of five of these cultivars, chosen on the basis of their salt tolerance, responded to $\mathrm{NaCl}$ in respect to root growth.

\section{Materials and Methods}

Seeds of 'Grand Rapids', 'Climax', 'Coolguard', 'Empire', 'Great Lakes 659-700', 'Mesa 659', 'Salinas', 'Vanguard 75', 'Red Coach 74', and 'Wintersupreme' lettuce were obtained from Asgrow Seed Co. (Kalamazoo, Mich.), SunSeeds (Hollister, Calif.), and Quali-Sel Seed (Salinas, Calif.). All are crisphead types except 'Grand Rapids', which is a leaf type.

Aqueous solutions of $\mathrm{NaCl}$ were prepared to obtain osmotic potentials of $0.0,-0.3,-0.6,-0.9,-1.2$, or $-1.5 \mathrm{MPa}$ $\left(0.0,3.5,7.1,10.6,14.2\right.$, or $17.7 \mathrm{~g} \cdot$ liter $^{-1}$, respectively). Osmotic potentials were checked on a vapor pressure osmometer (Wescor 5100 C, Logan, Utah).

Fifty seeds of each cultivar were placed in glass petri dishes $(10 \times 2 \mathrm{~cm})$, lined with three sheets of filter paper (Whatman $\# 1$ ), and moistened with $5 \mathrm{ml}$ of the appropriate $\mathrm{NaCl}$ solution. All dishes were placed inside clear plastic tubs (35 $x 24 \times 12$ $\mathrm{cm}$; Rubbermaid) to reduce water loss. No additional solution was added. Tubs were placed in growth chambers in the light for $12 \mathrm{hr}$ with $60 \mu \mathrm{mol} \cdot \mathrm{s}^{-1} \cdot \mathrm{m}^{-2}$ at $20,25,30$, or $35 \pm 2 \mathrm{C}$, as measured with thermistors. Two petri dishes per replication were used with three replications. 
Germinated seeds were counted every day for 14 days. Seeds were considered germinated when $1 \mathrm{~mm}$ of radicle was visible. Germination percentages after 14 days were calculated. Germination rate was calculated as the summation of newly germinated seeds on each day divided by number of days that elapsed since onset of imbibition with seed numbers adjusted to abase of 100 (Maguire, 1962). The highest possible theoretical value using this calculation is 100 , i.e., all seeds germinated on the 1st day.

Ten seeds from each of five cultivars selected on the basis of their salt tolerance in the germination experiments (sensitive'Great Lakes 659-700' and 'Mesa 659'; intermediate-'Climax'; tolerant-'Empire' and 'Vanguard 75') were placed in seed envelopes (Northrup King; $16.5 \times 17.5 \mathrm{~cm}$ ) that were moistened with $5 \mathrm{ml}$ of the appropriate $\mathrm{NaCl}$ solution. The envelopes were suspended on a frame in an upright position and placed in a lighted $\left(12 \mathrm{hr}\right.$ with $\left.60 \mu \mathrm{mol} \cdot \mathrm{s}^{-1} \cdot \mathrm{m}^{-2}\right)$ growth chamber held at $26 \pm 2 \mathrm{C}$. Every other day, an additional $5 \mathrm{ml}$ of the appropriate $\mathrm{NaCl}$ solution was added to replenish losses. Root length of each seedling was measured every other day for 14 days. Each cultivar- $\mathrm{NaCl}$ combination was replicated twice over time with two envelopes per replication.

For germination experiments, the experimental design was a three-factor split-plot experiment with the whole factor (temperature) arranged in a balanced incomplete-blocks design. An incomplete block consisted of one run with two growth chambers, each with one of the experimental temperatures. The subplot factors (cultivars and $\mathrm{NaCl}$ ) were arranged randomly within each of the whole plot temperature-growth chamber units. A balanced incomplete-blocks design analysis of variance (ANOVA) was conducted for germination percentage and rate. With the incomplete-blocks design it was necessary to calculate the least squares means adjusted for block effects (Cochran and Cox, 1957). Data on germination percentage were subjected to arcsin transformation prior to analysis. Least significant differences were calculated for mean separation at $P=0.05$.

For root length experiments, the design was a randomized complete block. Data were analyzed by a two-way ANOVA with the factors being cultivar and $\mathrm{NaCl}$. Means were separated by calculating least significant difference at $P=0.05$.

\section{Results}

Germination percentage. The ANOVA table for germination percentages on arcsin-transformed data showed that all factors and all interactions of factors were significant at $P=0.01$. Thus, data for various factors were considered separately (Table 1). With $0.0 \mathrm{MPa} \mathrm{NaCl}$, no significant differences in germination percentage were observed among cultivars at 20 or $25 \mathrm{C}$. At 30C, significant cultivar differences were observed, although all values were $94 \%$ or higher. These differences were even greater at 35C where none reached even $20 \%$. Germination percentages did not decrease significantly for each cultivar until $35 \mathrm{C}$, except for 'Coolguard' and 'Salinas', which had significantly lower germination at 30 than at $20 \mathrm{C}$.

At $-0.3 \mathrm{MPa} \mathrm{NaCl}$, significant cultivar differences still were not present for germination percentages at $20 \mathrm{C}$, but were at $25 \mathrm{C}$ (Table 1). At 35C, germination percentage dropped to near zero for all cultivars with no significant differences among them. Several more cultivars showed significant decreases in germination percentages at $30 \mathrm{C}$ relative to $20 \mathrm{C}$, i.e., 'Climax', 'Coolguard', 'Great Lakes 659-700', 'Mesa 659', 'Salinas', 'Red Coach 74', and 'Wintersupreme'. Thus, 'Grand Rapids', 'Empire', and 'Vanguard 75' were most tolerant to the higher tem-
Table 1. Least squares estimates of germination percentages after 14 days for 10 lettuce cultivars at four temperatures with five $\mathrm{NaCl}$ concentrations. ${ }^{2 . y}$

\begin{tabular}{|c|c|c|c|c|}
\hline \multirow[b]{2}{*}{ Cultivar } & \multicolumn{4}{|c|}{ Temperature $\left({ }^{\circ} \mathrm{C}\right)$} \\
\hline & 20 & 25 & 30 & 35 \\
\hline & \multicolumn{4}{|c|}{$0.0 \mathrm{MPa} \mathrm{NaCl}$} \\
\hline Grand Rapids & $100 \mathrm{a}$ & $97 \mathrm{a}$ & $94 \mathrm{c}$ & $9 \mathrm{~b}$ \\
\hline Climax & $100 \mathrm{a}$ & 99 a & $97 \mathrm{abc}$ & $0 \mathrm{c}$ \\
\hline Coolguard & $100 \mathrm{a}$ & $98 \mathrm{a}$ & $95 \mathrm{c}$ & $10 \mathrm{ab}$ \\
\hline Empire & $100 \mathrm{a}$ & $98 \mathrm{a}$ & $97 \mathrm{abc}$ & $19 a$ \\
\hline Great Lakes $659-700$ & $100 \mathrm{a}$ & $99 \mathrm{a}$ & $97 \mathrm{abc}$ & $0 \mathrm{c}$ \\
\hline Mesa 659 & $100 \mathrm{a}$ & $98 a$ & $96 \mathrm{bc}$ & $1 \mathrm{c}$ \\
\hline Salinas & $100 \mathrm{a}$ & $99 \mathrm{a}$ & $95 c$ & $0 \mathrm{c}$ \\
\hline Vanguard 75 & $100 \mathrm{a}$ & $99 \mathrm{a}$ & $99 \mathrm{ab}$ & $1 \mathrm{c}$ \\
\hline Red Coach 74 & $100 \mathrm{a}$ & $99 \mathrm{a}$ & $98 \mathrm{abc}$ & $18 \mathrm{a}$ \\
\hline \multirow[t]{2}{*}{ Wintersupreme } & $100 \mathrm{a}$ & 100 a & $99 \mathrm{a}$ & $9 \mathrm{~b}$ \\
\hline & \multicolumn{4}{|c|}{$-0.3 \mathrm{MPa} \mathrm{NaCl}$} \\
\hline Grand Rapids & $99 \mathrm{a}$ & $98 \mathrm{ab}$ & $95 \mathrm{ab}$ & $1 \mathrm{a}$ \\
\hline Climax & $99 \mathrm{a}$ & $99 \mathrm{a}$ & $91 \mathrm{bc}$ & $0 \mathrm{a}$ \\
\hline Coolguard & $100 \mathrm{a}$ & $97 \mathrm{ab}$ & $95 \mathrm{ab}$ & $3 a$ \\
\hline Empire & $100 \mathrm{a}$ & $98 \mathrm{ab}$ & $96 \mathrm{ab}$ & 1 a \\
\hline Great Lakes $659-700$ & $100 \mathrm{a}$ & $99 \mathrm{ab}$ & $71 \mathrm{e}$ & $0 \mathrm{a}$ \\
\hline Mesa 659 & $98 \mathrm{a}$ & $96 \mathrm{~b}$ & $80 \mathrm{de}$ & $0 \mathrm{a}$ \\
\hline Salinas & $100 \mathrm{a}$ & $98 \mathrm{ab}$ & $85 \mathrm{~cd}$ & $0 \mathrm{a}$ \\
\hline Vanguard 75 & $100 \mathrm{a}$ & $99 \mathrm{a}$ & $96 \mathrm{a}$ & $0 \mathrm{a}$ \\
\hline Red Coach 74 & $100 \mathrm{a}$ & $100 \mathrm{a}$ & $96 a b$ & $0 \mathrm{a}$ \\
\hline \multirow[t]{2}{*}{ Wintersupreme } & $100 \mathrm{a}$ & $99 \mathrm{ab}$ & $86 \mathrm{~cd}$ & $0 \mathrm{a}$ \\
\hline & \multicolumn{4}{|c|}{$-0.6 \mathrm{MPa} \mathrm{NaCl}$} \\
\hline Grand Rapids & $98 \mathrm{ab}$ & $95 \mathrm{~b}$ & $40 \mathrm{c}$ & 0 a \\
\hline Climax & $100 a b$ & $99 a$ & $36 \mathrm{c}$ & $0 \mathrm{a}$ \\
\hline Coolguard & $99 \mathrm{ab}$ & $97 \mathrm{ab}$ & $90 \mathrm{a}$ & $0 \mathrm{a}$ \\
\hline Empire & $100 \mathrm{ab}$ & $98 \mathrm{ab}$ & $79 \mathrm{~b}$ & $1 \mathrm{a}$ \\
\hline Great Lakes $659-700$ & $97 \mathrm{~b}$ & $98 \mathrm{ab}$ & $2 \mathrm{f}$ & $0 \mathrm{a}$ \\
\hline Mesa 659 & $97 \mathrm{ab}$ & $98 \mathrm{ab}$ & $6 \mathrm{e}$ & $0 \mathrm{a}$ \\
\hline Salinas & $99 \mathrm{ab}$ & $97 a b$ & $24 \mathrm{~d}$ & $0 \mathrm{a}$ \\
\hline Vanguard 75 & $100 \mathrm{ab}$ & 99 a & $69 \mathrm{c}$ & $0 \mathrm{a}$ \\
\hline Red Coach 74 & $100 \mathrm{a}$ & $99 \mathrm{a}$ & $49 \mathrm{c}$ & $0 \mathrm{a}$ \\
\hline \multirow[t]{2}{*}{ Wintersupreme } & $100 \mathrm{a}$ & $96 \mathrm{ab}$ & $19 \mathrm{~d}$ & 0 a \\
\hline & \multicolumn{4}{|c|}{$-0.9 \mathrm{MPa} \mathrm{NaCl}$} \\
\hline Grand Rapids & $97 \mathrm{ab}$ & $89 \mathrm{bc}$ & $1 \mathrm{~cd}$ & $0 \mathrm{a}$ \\
\hline Climax & 99 a & $70 \mathrm{~d}$ & $0 \mathrm{~d}$ & $0 \mathrm{a}$ \\
\hline Coolguard & $98 \mathrm{ab}$ & 97 a & $23 \mathrm{a}$ & $1 \mathrm{a}$ \\
\hline Empire & 99 a & $82 \mathrm{~cd}$ & $7 \mathrm{~b}$ & $0 \mathrm{a}$ \\
\hline Great Lakes $659-700$ & $97 \mathrm{ab}$ & $35 \mathrm{f}$ & $0 \mathrm{~d}$ & $0 \mathrm{a}$ \\
\hline Mesa 659 & $93 \mathrm{~b}$ & $53 \mathrm{e}$ & $0 \mathrm{~d}$ & $0 \mathrm{a}$ \\
\hline Salinas & 99 a & $95 \mathrm{ab}$ & $0 d$ & $0 \mathrm{a}$ \\
\hline Vanguard 75 & $100 \mathrm{a}$ & $97 a$ & $3 \mathrm{bc}$ & $0 \mathrm{a}$ \\
\hline Red Coach 74 & $100 \mathrm{a}$ & $83 \mathrm{~cd}$ & $0 \mathrm{~cd}$ & $0 \mathrm{a}$ \\
\hline \multirow[t]{2}{*}{ Wintersupreme } & $92 \mathrm{~b}$ & $23 \mathrm{f}$ & $0 \mathrm{~d}$ & $0 \mathrm{a}$ \\
\hline & \multicolumn{4}{|c|}{$-1.2 \mathrm{MPa} \mathrm{NaCl}$} \\
\hline Grand Rapids & $75 \mathrm{bc}$ & $8 \mathrm{bc}$ & $0 \mathrm{a}$ & $0 \mathrm{a}$ \\
\hline Climax & $24 \mathrm{e}$ & $3 \mathrm{de}$ & $0 \mathrm{a}$ & $0 \mathrm{a}$ \\
\hline Coolguard & $77 \mathrm{ab}$ & $25 \mathrm{a}$ & $0 \mathrm{a}$ & $0 \mathrm{a}$ \\
\hline Empire & $74 \mathrm{bc}$ & $6 \mathrm{~cd}$ & $0 \mathrm{a}$ & $0 \mathrm{a}$ \\
\hline Great Lakes $659-700$ & $3 \mathrm{~g}$ & $1 \mathrm{de}$ & $0 \mathrm{a}$ & $0 \mathrm{a}$ \\
\hline Mesa 659 & $3 \mathrm{~g}$ & $0 \mathrm{e}$ & $0 \mathrm{a}$ & $0 \mathrm{a}$ \\
\hline Salinas & $65 \mathrm{c}$ & $2 \mathrm{de}$ & $0 \mathrm{a}$ & $0 \mathrm{a}$ \\
\hline Vanguard 75 & 85 a & $21 \mathrm{ab}$ & $0 \mathrm{a}$ & $0 \mathrm{a}$ \\
\hline Red Coach 74 & $48 \mathrm{~d}$ & $1 \mathrm{e}$ & $0 \mathrm{a}$ & $0 \mathrm{a}$ \\
\hline Wintersupreme & $8 \mathrm{f}$ & $0 \mathrm{e}$ & $0 \mathrm{a}$ & $0 \mathrm{a}$ \\
\hline
\end{tabular}

${ }^{2}$ Least squares means adjusted for block effects; standard error for difference among temperatures within one cultivar and $\mathrm{NaCl}$ concentration is 4.7; standard error for difference among cultivars within one temperature and $\mathrm{NaCl}$ concentration is 4.6; mean separation within each column for a given $\mathrm{NaCl}$ concentration based on least significant difference at $P=0.05$ on arcsin-transformed data.

${ }^{y}$ None of the seeds germinated at $-1.5 \mathrm{MPa} \mathrm{NaCl}$. 
peratures with no significant decrease in germination percentages until 35C.

At - 0.6 $\mathrm{MPa} \mathrm{NaCl}$, cultivar differences in germination percentages were apparent at 20, 25, and 30C (Table 1), and were greatest at 30C. All cultivars showed a significant decrease in germination percentage at $30 \mathrm{C}$ relative to $20 \mathrm{C}$, and it was at or near $0 \%$ at $35 \mathrm{C}$ for all.

Cultivar differences in germination percentage with $-0.9 \mathrm{MPa}$ $\mathrm{NaCl}$ were apparent at 20, 25, and 30C (Table 1). However, significant decreases now found were between 20 and $25 \mathrm{C}$ for 'Grand Rapids', 'Climax', 'Empire', 'Great Lakes 659-700', 'Mesa 659', 'Red Coach 74',. and 'Wintersupreme'. Germination was absent or minimal at $35 \mathrm{C}$ for all cultivars.

With -1.2 $\mathrm{MPa} \mathrm{NaCl}$, cultivars differed in germination percentages only at 20 and 25C (Table 1); none germinated at 30 or $35 \mathrm{C}$. Significant decreases were noted for all cultivars held at $25 \mathrm{C}$ relative to those held at 20C, except for 'Great Lakes 659-700' and 'Mesa 659' because germination percentages of these cultivars already were very low at $20 \mathrm{C}$.

At $-1.5 \mathrm{MPa} \mathrm{NaCI}$, none of the cultivars germinated at 25, 30 , or $35 \mathrm{C}$ (data not shown). At 20C, germination percentages were between $1 \%$ and 5\%, with those of 'Empire' being significantly higher than for 'Climax', 'Great Lakes 659-700', 'Mesa 659', 'Salinas', and 'Wintersupreme' while the remaining four cultivars were intermediate. Only for 'Empire' was germination percentage significantly higher at $20 \mathrm{C}$ than at higher temperatures (i.e., 30 and $35 \mathrm{C}$ ).

Germination rate. The ANOVA tablehfor germination rates showed that all factors and all interactions of factors were significant at $P=0.01$ : Thus, data for the different factors were considered separately (Table 2). With no $\mathrm{NaCl}$, germination rates were similar for all cultivars at 20 or $25 \mathrm{C}$, although they did respond differently at 30 and $35 \mathrm{C}$. Rates were significantly lower at $35 \mathrm{C}$ relative to $20 \mathrm{C}$ for all cultivars except 'Salinas', which was lower at 30C.

With $-0.3 \mathrm{MPa} \mathrm{NaCl}$, cultivar differences now were found at 20 and $25 \mathrm{C}$, although at $35 \mathrm{C}$ all cultivars failed to germinate (Table 2). All cultivars but 'Coolguard' and 'Empire' now showed decreases in germination rates at $30 \mathrm{C}$ relative to $20 \mathrm{C}$. For ' $\mathrm{Cli}$ max' and 'Wintersupreme', rates were higher at 25 than at $20 \mathrm{C}$.

At - 0.6 MPa NaCl, cultivar differences were noted within each temperature except at $35 \mathrm{C}$, where none germinated (Table 2). Significant decreases were noted at $30 \mathrm{C}$ relative to $20 \mathrm{C}$ for rates of each cultivar except 'Coolguard', for which germination rates did not decrease until 35C. The germination rate of 'Great Lakes 659-700' actually decreased significantly at 25C and again at $30 \mathrm{C}$.

At - 0.9 $\mathrm{MPa} \mathrm{NaCl}$, differences among cultivars within each temperature were found only at 20 and $25 \mathrm{C}$, with rates $\leq 5$ at 30 and 35C (Table 2). Significant decreases were noted for rates of some cultivars at $25 \mathrm{C}$ relative to $20 \mathrm{C}$, i.e., 'Grand Rapids', 'Climax', and 'Empire'.

With - 1.2 $\mathrm{MPa} \mathrm{NaCl}$, no cultivar differences in rates were present at 25,30 , or $35 \mathrm{C}$, but all were low (Table 2). At 20C, the rate for 'Empire' was significantly higher than the rates for 'Great Lakes 659-700', 'Mesa 659', or ' Wintersupreme'. Rates were significantly higher at 20 than $35 \mathrm{C}$ for 'Grand Rapids', 'Coolguard', and 'Vanguard 75'. For 'Empire', rates at 20C were higher than at all other temperatures.

At $-1.5 \mathrm{MPa} \mathrm{NaCl}$, rates were $\leq 1$ for all cultivars and temperatures (data not shown).

Root length. The effects of salt and cultivar were significant at $P=0.05$, although the interaction was not significant. With
Table 2. Least squares estimates of germination rate of 10 lettuce cultivars at four temperatures with five $\mathrm{NaCl}$ concentrations for 14 days ${ }^{2 y,}$, .

\begin{tabular}{|c|c|c|c|c|}
\hline \multirow[b]{2}{*}{ Cultivar } & \multicolumn{4}{|c|}{ Temperature $\left({ }^{\circ} \mathrm{C}\right)$} \\
\hline & 20 & 25 & 30 & 35 \\
\hline & \multicolumn{4}{|c|}{$0.0 \mathrm{MPa} \mathrm{NaCl}$} \\
\hline Grand Rapids & $94 \mathrm{a}$ & $93 \mathrm{a}$ & $91 \mathrm{ab}$ & $2 b$ \\
\hline Climax & $95 \mathrm{a}$ & $98 \mathrm{a}$ & $95 \mathrm{a}$ & $0 \mathrm{~b}$ \\
\hline Coolguard & $97 \mathrm{a}$ & $96 a$ & $93 \mathrm{a}$ & $6 \mathrm{ab}$ \\
\hline Empire & $95 \mathrm{a}$ & $95 \mathrm{a}$ & 94 a & $13 a$ \\
\hline Great Lakes 659-700 & $98 \mathrm{a}$ & $98 a$ & $91 \mathrm{ab}$ & $0 \mathrm{~b}$ \\
\hline Mesa 659 & 94 a & $98 \mathrm{a}$ & $93 a$ & $0 \mathrm{~b}$ \\
\hline Salinas & $97 \mathrm{a}$ & 98 a & $82 \mathrm{~b}$ & $0 \mathrm{~b}$ \\
\hline Vanguard 75 & 99 a & $97 \mathrm{a}$ & $96 \mathrm{a}$ & $0 \mathrm{~b}$ \\
\hline Red Coach 74 & $100 \mathrm{a}$ & $98 \mathrm{a}$ & 98 a & $14 \mathrm{a}$ \\
\hline \multirow[t]{2}{*}{ Wintersupreme } & 92 a & $100 \mathrm{a}$ & 99 a & $6 \mathrm{ab}$ \\
\hline & \multicolumn{4}{|c|}{$-0.3 \mathrm{MPa} \mathrm{NaCl}$} \\
\hline Grand Rapids & $85 \mathrm{~b}$ & $94 \mathrm{ab}$ & $74 b$ & $0 \mathrm{a}$ \\
\hline Climax & $68 \mathrm{c}$ & $93 \mathrm{ab}$ & $62 \mathrm{~cd}$ & $0 \mathrm{a}$ \\
\hline Coolguard & $94 \mathrm{ab}$ & $93 \mathrm{abc}$ & 90 a & $0 \mathrm{a}$ \\
\hline Empire & $93 \mathrm{ab}$ & $96 \mathrm{ab}$ & $87 a$ & $0 \mathrm{a}$ \\
\hline Great Lakes 659-700 & $69 c$ & $70 \mathrm{~d}$ & $23 \mathrm{f}$ & $0 \mathrm{a}$ \\
\hline Mesa 659 & $70 \mathrm{c}$ & $65 d$ & $43 \mathrm{e}$ & $0 \mathrm{a}$ \\
\hline Salinas & $88 \mathrm{ab}$ & $87 \mathrm{bc}$ & $54 \mathrm{~d}$ & $0 \mathrm{a}$ \\
\hline Vanguard 75 & $96 \mathrm{a}$ & $96 \mathrm{ab}$ & $67 \mathrm{bc}$ & $0 \mathrm{a}$ \\
\hline Red Coach 74 & $93 \mathrm{ab}$ & $100 \mathrm{a}$ & $70 \mathrm{bc}$ & $0 \mathrm{a}$ \\
\hline \multirow[t]{2}{*}{ Wintersupreme } & $72 \mathrm{c}$ & $83 c$ & $54 \mathrm{~d}$ & $0 \mathrm{a}$ \\
\hline & \multicolumn{4}{|c|}{$-0.6 \mathrm{MPa} \mathrm{NaCl}$} \\
\hline Grand Rapids & $50 \mathrm{bcd}$ & $57 \mathrm{~b}$ & $18 \mathrm{~cd}$ & $0 \mathrm{a}$ \\
\hline Climax & $46 \mathrm{~cd}$ & $51 \mathrm{bc}$ & $8 \mathrm{def}$ & $0 \mathrm{a}$ \\
\hline Coolguard & $65 \mathrm{a}$ & $70 \mathrm{a}$ & $63 \mathrm{a}$ & $0 \mathrm{a}$ \\
\hline Empire & $66 \mathrm{a}$ & $67 a$ & $35 \mathrm{~b}$ & $0 \mathrm{a}$ \\
\hline Great Lakes 659-700 & $44 \mathrm{de}$ & $28 \mathrm{~d}$ & $1 \mathrm{f}$ & $0 \mathrm{a}$ \\
\hline Mesa 659 & $42 \mathrm{de}$ & $33 \mathrm{~d}$ & $3 \mathrm{f}$ & $0 \mathrm{a}$ \\
\hline Salinas & 45 cde & $46 c$ & $7 \mathrm{ef}$ & $0 \mathrm{a}$ \\
\hline Vanguard 75 & $54 \mathrm{bc}$ & $56 \mathrm{~b}$ & $25 c$ & $0 \mathrm{a}$ \\
\hline Red Coach 74 & $56 \mathrm{ab}$ & $52 \mathrm{bc}$ & 15 cde & $0 \mathrm{a}$ \\
\hline \multirow[t]{2}{*}{ Wintersupreme } & $36 \mathrm{e}$ & $32 \mathrm{~d}$ & $6 \mathrm{ef}$ & $0 \mathrm{a}$ \\
\hline & \multicolumn{4}{|c|}{$-0.9 \mathrm{MPa} \mathrm{NaCl}$} \\
\hline Grand Rapids & $32 \mathrm{~b}$ & $22 \mathrm{a}$ & $1 \mathrm{a}$ & $0 \mathrm{a}$ \\
\hline Climax & $19 \mathrm{cde}$ & $7 \mathrm{c}$ & $1 \mathrm{a}$ & $0 \mathrm{a}$ \\
\hline Coolguard & $28 \mathrm{bc}$ & $26 a$ & 5 a & 0 a \\
\hline Empire & $42 \mathrm{a}$ & $20 \mathrm{ab}$ & $2 \mathrm{a}$ & 0 a \\
\hline Great Lakes 659-700 & $14 \mathrm{de}$ & $4 c$ & $1 \mathrm{a}$ & $0 \mathrm{a}$ \\
\hline Mesa 659 & 14 de & $5 \mathrm{c}$ & $1 \mathrm{a}$ & $0 \mathrm{a}$ \\
\hline Salinas & $22 \mathrm{bcd}$ & $20 \mathrm{ab}$ & $1 \mathrm{a}$ & $0 \mathrm{a}$ \\
\hline Vanguard 75 & $26 \mathrm{bc}$ & $29 a$ & $2 a$ & $0 \mathrm{a}$ \\
\hline Red Coach 74 & $19 \mathrm{cde}$ & $11 \mathrm{bc}$ & $1 \mathrm{a}$ & $0 \mathrm{a}$ \\
\hline \multirow[t]{2}{*}{ Wintersupreme } & $12 \mathrm{e}$ & $3 \mathrm{c}$ & $1 \mathrm{a}$ & $0 \mathrm{a}$ \\
\hline & \multicolumn{4}{|c|}{$-1.2 \mathrm{MPa} \mathrm{NaCl}$} \\
\hline Grand Rapids & $10 \mathrm{ab}$ & $2 \mathrm{a}$ & $1 \mathrm{a}$ & $0 \mathrm{a}$ \\
\hline Climax & $3 a b$ & $3 a$ & $1 \mathbf{a}$ & $0 \mathrm{a}$ \\
\hline Coolguard & $9 \mathrm{ab}$ & $4 a$ & $1 \mathrm{a}$ & $0 \mathrm{a}$ \\
\hline Empire & $12 \mathrm{a}$ & $2 a$ & $1 \mathrm{a}$ & $0 \mathrm{a}$ \\
\hline Great Lakes $659-700$ & $1 \mathrm{~b}$ & $0 \mathrm{a}$ & $1 \mathrm{a}$ & $0 \mathrm{a}$ \\
\hline Mesa 659 & $1 \mathrm{~b}$ & $0 \mathrm{a}$ & $1 \mathrm{a}$ & $0 \mathrm{a}$ \\
\hline Salinas & $7 \mathrm{ab}$ & $1 \mathrm{a}$ & $1 \mathrm{a}$ & $0 \mathrm{a}$ \\
\hline Vanguard 75 & $10 \mathrm{ab}$ & $2 \mathrm{a}$ & $1 \mathrm{a}$ & $0 \mathrm{a}$ \\
\hline Red Coach 74 & $5 a b$ & $3 \mathrm{a}$ & $1 \mathrm{a}$ & $0 \mathrm{a}$ \\
\hline Wintersupreme & $1 \mathrm{~b}$ & $1 \mathrm{a}$ & $1 \mathrm{a}$ & $0 \mathrm{a}$ \\
\hline
\end{tabular}

${ }^{2}$ Germination rate: summation of newly germinated seeds on each day divided by number of days that elapsed since onset of imbibition.

'Least squares means adjusted for block effects; standard error for difference among temperatures within one cultivar and $\mathrm{NaCl}$ concentration is 5.1; standard error for difference among cultivars within one temperature and $\mathrm{NaCl}$ concentration is 4.9; mean separation within each column for a given $\mathrm{NaCl}$ concentration based on least significant difference at $\boldsymbol{P}=0.05$. ${ }^{\times}$None of the seeds germinated at $-1.5 \mathrm{MPa} \mathrm{NaCl}$. 
0.0 MPa NaCl, 'Empire' and 'Great Lakes 659-700' had significantly longer roots than other cultivars (Table 3). At -0.3 $\mathrm{MPa} \mathrm{NaCl}$, root lengths for 'Empire' and 'Great Lakes 659700 ' still were similar but only those of 'Empire' were longer than those of all other cultivars. With -0.6 and $-0: 9 \mathrm{MPa}$ $\mathrm{NaCl}$, 'Great Lakes 659-700' and 'Mesa 659' had no root growth and 'Climax' nearly none. At - 1.2 MPa NaCl, only 'Empire' still grew roots. No root growth occurred for any cultivars at $-1.5 \mathrm{MPa} \mathrm{NaC} 1$. Thus, sensitivity to $\mathrm{NaCl}$ based on root length was greatest for 'Great Lakes 659-700' and 'Mesa 659', intermediate for 'Climax', and least for 'Empire' and 'Vanguard 75'. For 'Empire' and 'Vanguard 75', roots were longer at -0.3 $\mathrm{MPa}$ than at $0.0 \mathrm{MPa}$.

\section{Discussion}

Cultivar differences in germination were observed in response to high temperatures. With no $\mathrm{NaCl}$, differences between sensitive and tolerant cultivars were not as clear as they were with NaCl. 'Coolguard', 'Empire', 'Red Coach 74', and to some extent 'Wintersupreme' were tolerant to high temperature relative to 'Climax', 'Great Lakes 659-700', 'Mesa 659', 'Salinas', and 'Vanguard 75'. However, these groupings narrowed considerably as $\mathrm{NaCl}$ was added. With high temperature and $\mathrm{NaCl}$, 'Coolguard' and 'Empire' were clearly the most tolerant cultivars, and to some extent 'Grand Rapids' and 'Vanguard 75' were tolerant. The most sensitive cultivars with high temperature and $\mathrm{NaCl}$ clearly were 'Great Lakes 659-700' and 'Mesa 659'. Other studies comparing lettuce cultivars for sensitivity to salt used many cultivars other than those in the present study, although a few were the same. 'Climax' and 'Salinas' had similar salt tolerance (Pasternak et al., 1986), whereas 'Climax' had greater salt tolerance than 'Grand Rapids' (Shannon and McCreight, 1984). These discrepancies from findings in the present study may be related to their evaluation of salt tolerance by fresh weight at later stages or because greenhouse and field conditions provided less temperature control than our tests.

The maximum temperature for germination of lettuce varied depending upon cultivars and $\mathrm{NaCl}$ concentration. With no $\mathrm{NaCl}$, high temperature effects first were observed at 35C for all cultivars except 'Salinas', which showed significantly lower germination percentages and rates at 30 than at 20C. The temperatures where germination decreased significantly were similar to those reported for crisphead lettuce types in other studies (Gray, 1975), although 'Grand Rapids' is the only cultivar tested in the present and in previous studies (Borthwick and Robbins, 1928; Thompson et al., 1979). With the addition of $\mathrm{NaCl}$, the maximum germination temperatures were lower, as indicated by germination percentages and rates.

Table 3. Root lengths (centimeters) of five cultivars of lettuce seedlings held 14 days at $26 \mathrm{C}$ with five $\mathrm{NaCl}$ concentrations."

\begin{tabular}{lccccc}
\hline & \multicolumn{5}{c}{$\mathrm{NaCl}$ concn $(\mathrm{MPa})^{\mathrm{y}}$} \\
\cline { 2 - 6 } Cultivar & 0.0 & -0.3 & -0.6 & -0.9 & -1.2 \\
\hline Empire & $6.8 \mathrm{a}$ & $7.6 \mathrm{a}$ & $3.4 \mathrm{a}$ & $1.2 \mathrm{ab}$ & $1.0 \mathrm{a}$ \\
Vanguard 75 & $5.5 \mathrm{~b}$ & $6.6 \mathrm{bc}$ & $2.2 \mathrm{~b}$ & $1.4 \mathrm{a}$ & $0.0 \mathrm{~b}$ \\
Climax & $5.7 \mathrm{~b}$ & $5.9 \mathrm{c}$ & $2.5 \mathrm{~b}$ & $0.5 \mathrm{bc}$ & $0.0 \mathrm{~b}$ \\
Great Lakes 659-700 & $7.4 \mathrm{a}$ & $7.1 \mathrm{ab}$ & $0.0 \mathrm{c}$ & $0.0 \mathrm{c}$ & $0.0 \mathrm{~b}$ \\
Mesa 659 & $5.8 \mathrm{~b}$ & $6.5 \mathrm{bc}$ & $0.0 \mathrm{c}$ & $0.0 \mathrm{c}$ & $0.0 \mathrm{~b}$ \\
\hline
\end{tabular}

${ }^{2}$ None of the cultivars produced roots at $-1.5 \mathrm{MPa} \mathrm{NaCl}$.

${ }^{y}$ Mean separation within each column based on least significant difference at $P=0.05$; standard error for difference among $\mathrm{NaCl}$ concentrations within a cultivar is 0.38 ; standard error for difference between cultivars within a $\mathrm{NaCl}$ concentration is 0.35 .
The experiment on root lengths with a subset of cultivars confirmed our germination findings of relative tolerance to $\mathrm{NaCl}$ in some cultivars. For 'Great Lakes 659-700' and 'Mesa 659', which were rated sensitive at germination, the roots did not grow at all with $\mathrm{NaCl}$ less than or equal to $-0.6 \mathrm{MPa}$. This lack of root growth would prevent establishment of seedlings. 'Climax' was intermediate in tolerance to salt in both germination and root length studies. 'Empire' and 'Vanguard 75' clearly were tolerant relative to the other cultivars in root length studies where root growth in length actually was stimulated at $-0.3 \mathrm{MPa} \mathrm{NaCl}$ relative to no $\mathrm{NaCl}$. In the germination studies, "'Empire' clearly was tolerant, and 'Vanguard 75' was tolerant to some extent. The tolerant cultivars would produce more vigorous roots than sensitive ones in the presence of $\mathrm{NaCl}$, and thus establish a better stand.

From a "grower standpoint, if soil temperatures are near or above $35 \mathrm{C}$ all day, germination, problems may develop for the cultivars tested (mostly crisphead types) even if no salts (i.e., $\mathrm{NaCl}$ ) were present in the soil or irrigation water. However, the presence of $\mathrm{NaCl}$ would accentuate this high temperature problem. If $\mathrm{NaCl}$ is present, extremely sensitive cultivars such as 'Great Lakes 659-700' and 'Mesa 659' should be avoided. Cultivars that are more tolerant to high temperature and $\mathrm{NaCl}$ included 'Coolguard', 'Empire', and to some extent 'Grand Rapids' and 'Vanguard 75'. Consideration should be given to the concentration of salt both in the irrigation water and in the upper few centimeters of soil where lettuce seeds are placed.

\section{Literature Cited}

Ayers, A. D., C.H. Wadleigh, and L. Bernstein. 1951. Salt tolerance of six varieties of lettuce. Proc. Amer. Soc. Hort. Sci. 57:237-242.

Borthwick, H.A. and W.W. Robbins. 1928. Structure of lettuce seed as related to germination. Hilgardia 3:275-305.

Cochran, W.G. and G.M. Cox. 1957. Experimental designs. 2nd ed. Wiley, New York.

Dutt, G. R., and T.W. McCreary. 1970. The quality of Arizona's domestic, agricultural, and industrial waters. Report 256. Agr. Expt. Sta., Univ. of Arizona.

Gray, D. 1975. Effects of temperature on the germination and emergence of lettuce (Lactuca sativa L.) varieties. J. Hort. Sci. 50:349-361.

Krause, W. 1980. Crisp and even: Can it be done? Grower 94:53-54.

Maas, E.V. and G.F. Hoffman. 1977. Crop salt tolerance: Evaluation of existing data. Proc. Intl. Salinity Conf. Texas Tech. Univ., Lubbock, Texas. Aug. 1976:187-198.

Maguire, J.D. 1962. Speed of germination-aid in selection and evaluation for seedling emergence and vigor. Crop Sci. 2:176-177.

Odegbaro, O.A. and O.E. Smith. 1969. Effects of kinetin, salt concentration, and temperature on germination and early seedling growth of Lactuca sativa L. J. Amer. Soc. Hort. Sci. 94:167-170.

Pasternak, D., Y. DeMalach, I. Borovic, M. Shram, and C. Aviram. 1986. Irrigation with brackish water under desert conditions, IV. Salt tolerance studies with lettuce (Lactuca sativa L.). Agr. Water Mgt. 11:303-311.

Shannon, M.C. and J.D. McCreight. 1984. Salt tolerance of lettuce introductions. HortScience 19:673-675.

Shannon, M. C., J.D. McCreight, and J.H. Draper. 1983. Screening tests for salt tolerance in lettuce. J. Amer. Soc. Hort. Sci. 108:225-230.

Thompson, P. A., S.A. Cox, and R.H. Sanderson. 1979. Characterization of the germination responses to temperature of lettuce (Latuca sativa L.) achenes. Ann. Bet. 43:319-334.

Valdes, V.M. and K.J. Bradford. 1987. Effects of seed coating and osmotic priming on the germination of lettuce seeds. J. Amer. Soc. Hort. Sci. 112:153-156.

Wurr, D.C.E. and J.R. Fellows. 1983. The effect of the time of seedling emergence of crisp lettuce on the time of maturity and head weight at maturity. J. Hort. Sci. 58:561-566. 